\title{
Designing the mesoscopic approach of an autonomous linear dynamical system by a quantum formulation
}

\author{
Micó, Joan C.
}

Departament de Matemàtica Aplicada, Universitat Politècnica de Valéncia, Spain.jmico@mat.upv.es

\begin{abstract}
This paper is an attempt to translate the quantum formulation from physics to general systems modelled by dynamical systems. Their quantum formulation provides a mesoscopic approach due to the stochastic nature of quantum theory. A quantum formulation needs a previous Hamiltonian. A first order Hamiltonian was provided in past works by following Dirac's generalized dynamics. The corresponding quantum approach, given by a first order Schrodinger equation, was provided from the Hamiltonian found, by applying the corresponding quantization rules. The split of the wave function in its amplitude and phase provides the probability conservation law for the square amplitude and the HamiltonJacobi equation for the phase. However, this last equation lacks the stochastic term, in opposition to the stochastic term appearing for the current Schrodinger equation corresponding to the physics laws. Thus, the approach presented in past works is unsuitable to obtain a mesoscopic approach for dynamical systems. The hypothesis here presented considers the existence of a second order Hamiltonian from the base that the physics laws can be defined with a similar structure. The existence of a Hamiltonian like this is proved to be always possible for the case of an autonomous linear dynamical system. In the beginning the Schrodinger equation is written for this case, as well as its timeindependent version. This case does present a mesoscopic approach, which is developed and its stochastic term is stressed. The general solution of the Schrodinger equation is found and two application cases are presented. In the conclusions section some ways are sketched about how to generalize the formalism to nonlinear dynamical systems.
\end{abstract}

Keywords: linear autonomous dynamical system, Hamiltonian, Hamilton-Jacobi equation, Schrodinger equation, mesoscopic approach.

\section{Introduction}

The objective of this paper is to present a quantum formulation for dynamical systems and to deduce from it a mesoscopic approach for this kind of systems. Note that the definition of dynamical sytem here 
considered is a coupled set of first order differential equations with the time as independent variable. In addition, "mesoscopic" means "deterministic" plus "stochatic".

Take into account that this kind of systems relate dynamically variables of different nature, in opposition to the dynamical systems studied in physics whose nature is spatial-related. The first systems are also referred here as general systems and the second systems are also referred as natural systems. In addition, from a pure mathematical point of view, the natural systems are coupled sets of second order differential equations with the time as independent variable, in opposition to the general systems here considered with a first order differential structure.

Besides, the Hamiltonian of a natural system permits, trough the quantization rules of the Copenhagen formalism (Galindo \& Pascual, 1990), getting the corresponding Schrodinger equation. In addition, from the polar form of the wave function is eassy to deduce that: (a) the probability is conserved for the square amplitude of the wave function; (b) the phase holds the Hamilton-Jacobi equations except a stochastic term. The stochastic term can be introduced into the Hamiltonian and, from it, to the previous dynamical equations. These new equations present the old deterministic term plus a stochastic term that depends on the amplitude of the wave function, i.e, the mesoscopic approach to a natural system.

The same program of research described for natural systems in physics is here broutgt for general systems as an attempt to state an isomorphism between physics and general systems theory. Depenning in the formalism, the approach here presented starts from a deterministic general system without a stochastic term. In order to reach a quantum formulation a Hamiltonian is needed on which applies the quantization rules of the Copenhagen formalism. The first Hamiltonian provided is first order in momenta (first order Hamiltonian from now onwards), provided by Pontryagin (1985), although other similar first order Hamiltonians can be provided following Hava's method (1973) or Dirac's generalized dynamics (1964). In the beginning the quantization rules are applied, arising a first order Schrodinger equation (Micó, 2014a; Micó, 2014b). However, the polar split of the first order Schrodinger equation does not present a stochastic term for the Hamilton-Jacobi equation, and a mesoscopic approach is not possible with this approach (Micó, 2014b).

The option followed in this paper is trying to obtain a second order Hamiltonian in momenta (second order Hamiltonian from now onwards) that provides a second order Schrodinger equation. Only if some conditions hold the functions that define the general system is possible to obtain a second order Hamiltonian. In fact, the application cases given by the one-dimensional systems and by the autonomous linear systems hold always those conditions. This last case is what provides the title of the paper. In addition, it can be seen that this second order Schrodinger equation does provide the stochastic term in the corresponding Hamilton-Jacobi equation.

Moreover, the present approach is compared with the approach presented by Haken (2004) through the Fokker-Planck equation, for which the starting point is a stochastic differential system, i.e., the Ito equations. These equations present the stochastic terms in advance, and from them, the Fokker-Planck equation is derived, which would be equivalent to a real (non complex) second order Schrodinger equation.

The presentation of the paper contents are the following. Section 2 is devoted to the revision of the first order Hamiltonian and its corresponding first order Schrodinger equation. Section 3 provides the structure of a second order Hamiltonian and the conditions for its existence. The second order Schrodinger equation and its probabilistic interpretation is provided in Section 4. The mesoscopic approach derived and its comparison with Haken's approach is presented in Section 5. Section 6 is devoted to the 
application cases: the one-dimensional systems and the autonomous linear systems. The paper discussion and the paper conclusions are presented in Section 7.

\section{First order Hamiltonian and first order Schrodinger equation}

Let $q_{k}(t), k=1,2, \ldots, n$, be the state variables of a general system, being $n$ the system dimensions, with $\boldsymbol{q}=\left(q_{1}, q_{2}, \ldots, q_{n}\right)$ :

$$
\dot{q}_{k}(t)=f_{k}(t, \boldsymbol{q})(1)
$$

From now onwards every subscript will vary from 1 to $n$. Pontryagin's approach (Pontryagin, 1985) provides the eassiest first order Hamiltonian in momenta to (1):

$$
H(t, \boldsymbol{q}, \boldsymbol{p})=V(t, \boldsymbol{q})+\sum_{j} f_{j}(t, \boldsymbol{q}) \cdot p_{j}(2)
$$

where $\boldsymbol{p}=\left(p_{1}, p_{2}, \ldots, p_{n}\right)$ are the canonical momenta and $V(t, \boldsymbol{q})$ is a known function to be optimized. In Dirac's generalized dynamics (Dirac, 1964) $V(t, \boldsymbol{q})$ is deduced from the formalism as well as in Hava's approach (Havas, 1973). See (Micó, 2014a) and (Micó, 2014b) for the details of both approaches. In the subsequent development of the theory, this term is considered zero, because no function has to be optimized. Thus, the corresponding canonical equations, with $V(t, \boldsymbol{q})=0$, are:

$$
\begin{gathered}
\dot{q}_{k}=\frac{\partial H(t, \boldsymbol{q}, \boldsymbol{p})}{\partial p_{k}}=f_{k}(t, \boldsymbol{q})(3) \\
\dot{p}_{k}=-\frac{\partial H(t, \boldsymbol{q}, \boldsymbol{p})}{\partial q_{k}}=-\sum_{j} \frac{\partial f_{j}(t, \boldsymbol{q})}{\partial q_{k}} p_{j}(4)
\end{gathered}
$$

Then (3) holds (1). The Hamilton-Jacobi equation is a partial differential equation for the action $S(t, \boldsymbol{q})$, corresponding to the Hamiltonian (2), which can be written by using the vector notation, $\boldsymbol{f}(t, \boldsymbol{q})=$ $\left(f_{1}(t, \boldsymbol{q}), \ldots, f_{n}(t, \boldsymbol{q})\right), \boldsymbol{\nabla}=\left(\frac{\partial}{\partial q_{1}}, \ldots, \frac{\partial}{\partial q_{n}}\right):$

$$
\frac{\partial S(t, \boldsymbol{q})}{\partial t}+\boldsymbol{f}(t, \boldsymbol{q}) \nabla S(t, \boldsymbol{q})=0(5)
$$

To find the Schrodinger equation corresponding to the Hamiltonian (2), the quantization rules provided for the Copenhagen formalism of the quantum theory must be followed (Galindo \& Pascual, 1990). The formalism points out that the Hamiltonian becomes an operator, $\widehat{H}(t, \widehat{\boldsymbol{q}}, \widehat{\boldsymbol{p}})$, such that it operates on the wave function $\Psi(t, \boldsymbol{q})$ as:

$$
\widehat{H}(t, \widehat{\boldsymbol{q}}, \widehat{\boldsymbol{p}}) \Psi(t, \boldsymbol{q})=\frac{1}{2}\left(f_{i}(t, \widehat{\boldsymbol{q}}) \hat{p}_{i}+\hat{p}_{i} f_{i}(t, \widehat{\boldsymbol{q}})\right) \Psi(t, \boldsymbol{q})(6)
$$


such that:

$$
\begin{aligned}
f_{i}(t, \widehat{\boldsymbol{q}}) \Psi(t, \boldsymbol{q}) & =f_{i}(t, \boldsymbol{q}) \Psi(t, \boldsymbol{q})(7) \\
\hat{p}_{i} \Psi(t, \boldsymbol{q}) & =-i \sigma \frac{\partial \Psi(t, \boldsymbol{q})}{\partial q_{i}}(8)
\end{aligned}
$$

being $\sigma$ the system Planck constant, which in the present approach is considered hypotetically particular of each system and not coinciding with the Planck constant of physics. Another hypothesis about the system Planck constant is that the particular value represents a limitation of the mathematical knowledge of the system. However, this hypothesis has not been yet demonstrated.

From (7) and (8) in (6), using again the vector notation, and after some computations:

$$
\widehat{H}(t, \widehat{\boldsymbol{q}}, \widehat{\boldsymbol{p}}) \Psi(t, \boldsymbol{q})=-i \sigma \boldsymbol{f}(t, \boldsymbol{q}) \boldsymbol{\nabla} \Psi(t, \boldsymbol{q})-i \frac{\sigma}{2}(\boldsymbol{\nabla} \boldsymbol{f}(t, \boldsymbol{q})) \Psi(t, \boldsymbol{q})(9)
$$

Therefore, following the quantization rules (Galindo \& Pascual, 1990) the Schrödinger equation is written as:

$$
i \sigma \frac{\partial \Psi(t, \boldsymbol{q})}{\partial t}=\widehat{H}(t, \widehat{\boldsymbol{q}}, \widehat{\boldsymbol{p}}) \Psi(t, \boldsymbol{q})(10)
$$

The substitution of (9) in (10) provides:

$$
i \sigma \frac{\partial \Psi(t, \boldsymbol{q})}{\partial t}=-i \sigma \boldsymbol{f}(t, \boldsymbol{q}) \boldsymbol{\nabla} \Psi(t, \boldsymbol{q})-i \frac{\sigma}{2}(\boldsymbol{\nabla} \boldsymbol{f}(t, \boldsymbol{q})) \Psi(t, \boldsymbol{q})(11)
$$

Equation (11) is the first order Schrödinger equation corresponding to the general system (1). Its probabilistic interpretation can be done through the split of the wave function in polar coordinates:

$$
\Psi(t, \boldsymbol{q})=\mathrm{A}(t, \boldsymbol{q}) \mathrm{e}^{i \frac{\mathrm{B}(t, \boldsymbol{q})}{\sigma}}
$$

where $\mathrm{A}(t, \boldsymbol{q})$ is the amplitude and $\mathrm{B}(t, \boldsymbol{q})$ is the phase. The substitution of (12) in (11) provides, respectively for real and the imaginary parts of the equation (after cancelling the term $\mathrm{e}^{i \frac{\mathrm{B}(t, \boldsymbol{q})}{\sigma}}$ ):

$$
-\mathrm{A}(t, \boldsymbol{q}) \frac{\partial \mathrm{B}(t, \boldsymbol{q})}{\partial t}=\mathrm{A}(t, \boldsymbol{q}) \boldsymbol{f}(t, \boldsymbol{q}) \boldsymbol{\nabla B}(t, \boldsymbol{q})(13)
$$




$$
-\sigma \frac{\partial \mathrm{A}(t, \boldsymbol{q})}{\partial t}=-\sigma \boldsymbol{f}(t, \boldsymbol{q}) \boldsymbol{\nabla} \mathrm{A}(t, \boldsymbol{q})-\frac{\sigma}{2} \mathrm{~A}(t, \boldsymbol{q})(\boldsymbol{\nabla} \boldsymbol{f}(t, \boldsymbol{q}))(14)
$$

Dividing (13) by $\mathrm{A}(t, \boldsymbol{q})$ and dividing (14) by $\sigma$ and subsequently multiplying it by $2 \mathrm{~A}(t, \boldsymbol{q})$ :

$$
\begin{gathered}
\frac{\partial \mathrm{B}(t, \boldsymbol{q})}{\partial t}+\boldsymbol{f}(t, \boldsymbol{q}) \boldsymbol{\nabla B}(t, \boldsymbol{q})=0 \\
\frac{\partial \mathrm{A}^{2}(t, \boldsymbol{q})}{\partial t}+\boldsymbol{\nabla}\left(\boldsymbol{f}(t, \boldsymbol{q}) \mathrm{A}^{2}(t, \boldsymbol{q})\right)=0(16)
\end{gathered}
$$

Equation (15) provides the same Hamilton-Jacobi equation (5) for $\mathrm{B}(t, \boldsymbol{q})$, and Equation (16) provides the probability conservation of $\mathrm{A}^{2}(t, \boldsymbol{q})$, being $\boldsymbol{f}(t, \boldsymbol{q})$ the corresponding probability current density. Note that, contrary to the natural systems, a stochastic term in (15) that could difference it of (5) does not arise in this context. The Schrödinger equation (11) seems to be a deterministic approach to the general systems rather than a stochastic one. Therefore, it can not be a good mesoscopic approach to (1).

\section{Second order Hamiltonian}

In order to find a second order Hamiltonian, Equation (1) must become a second order system of differential equations. It is possible by taking the time derivative at the two sides of (1):

$$
\ddot{q}_{k}(t)=\dot{f}_{k}(t, \boldsymbol{q})=\frac{\partial f_{k}(t, \boldsymbol{q})}{\partial t}+\sum_{l} \frac{\partial f_{k}(t, \boldsymbol{q})}{\partial q_{l}} \dot{q}_{l}=\frac{\partial f_{k}(t, \boldsymbol{q})}{\partial t}+\sum_{l} \frac{\partial f_{k}(t, \boldsymbol{q})}{\partial q_{l}} f_{l}(t, \boldsymbol{q})(17)
$$

And the new Hamiltonian posed is:

$$
H(t, \boldsymbol{q}, \boldsymbol{p})=\frac{1}{2} \sum_{j l} u_{j l} p_{j} p_{l}+\sum_{j} f_{j}(t, \boldsymbol{q}) \cdot p_{j}(18)
$$

where $u_{j l}$ is a constant simetric matrix, i.e., a matrix with every element contant such that $u_{j l}=u_{l j}$. The problem now is searching the elements of $u_{j l}$ in (18) such that the canonical equations hold (17). Thus, applying those equations on (18):

$$
\begin{gathered}
\dot{q}_{k}=\frac{\partial H(t, \boldsymbol{q}, \boldsymbol{p})}{\partial p_{k}}=f_{k}(t, \boldsymbol{q})+\sum_{l} u_{k l} p_{l}(19) \\
\dot{p}_{k}=-\frac{\partial H(t, \boldsymbol{q}, \boldsymbol{p})}{\partial q_{k}}=-\sum_{j} \frac{\partial f_{j}(t, \boldsymbol{q})}{\partial q_{k}} p_{j}(20)
\end{gathered}
$$

The corresponding Hamilton-Jacobi equation of the Hamiltonian (18) for the action $S(t, \boldsymbol{q})$, is: 
Designing the mesoscopic approach of an autonomous linear dynamical system by a quantum formulation.

$$
\frac{\partial S(t, \boldsymbol{q})}{\partial t}+\frac{1}{2} \sum_{j l} u_{j l} \frac{\partial S(t, \boldsymbol{q})}{\partial q_{j}} \frac{\partial S(t, \boldsymbol{q})}{\partial q_{l}}+\boldsymbol{f}(t, \boldsymbol{q}) \nabla S(t, \boldsymbol{q})=0(21)
$$

Note that if the general system is autonomous, i.e., no function of (1) depends on the time, the same happens with the functions of (17), which becomes:

$$
\ddot{q}_{k}(t)=\dot{f}_{k}(\boldsymbol{q})=\sum_{l} \frac{\partial f_{k}(\boldsymbol{q})}{\partial q_{l}} f_{l}(\boldsymbol{q})(22)
$$

Moreover, in the autonomous case, the Hamiltonian does not depend explicitly on the time and it becomes a constant of the dynamics, wich coincides with the system energy $E$, i.e.:

$$
E=\frac{1}{2} \sum_{j l} u_{j l} p_{j} p_{l}+\sum_{j} f_{j}(\boldsymbol{q}) \cdot p_{j}(23)
$$

To obtain the values of the matrix $u_{j l}$ in (18), Equations (19) and (20) must be compared with (17). To do this, the time derivative is taked at the two sides of (19):

$$
\ddot{q}_{k}(t)=\frac{\partial f_{k}(t, \boldsymbol{q})}{\partial t}+\sum_{l} \frac{\partial f_{k}(t, \boldsymbol{q})}{\partial q_{l}} \dot{q}_{l}+\sum_{l} u_{k l} \dot{p}_{l}(24)
$$

Substituting respectively $\dot{q}_{l}$ and $\dot{p}_{l}$ from (19) and (20) in (24) and regrouping after some calculations:

$$
\ddot{q}_{k}(t)=\frac{\partial f_{k}(t, \boldsymbol{q})}{\partial t}+\sum_{l} \frac{\partial f_{k}(t, \boldsymbol{q})}{\partial q_{l}} f_{l}(t, \boldsymbol{q})+\sum_{j} p_{j}\left(\sum_{l} u_{j l} \frac{\partial f_{k}(t, \boldsymbol{q})}{\partial q_{l}}-\sum_{l} u_{k l} \frac{\partial f_{j}(t, \boldsymbol{q})}{\partial q_{l}}\right)
$$

Thus, in order that (25) and (17) coincide:

$$
\sum_{l} u_{j l} \frac{\partial f_{k}(t, \boldsymbol{q})}{\partial q_{l}}-\sum_{l} u_{k l} \frac{\partial f_{j}(t, \boldsymbol{q})}{\partial q_{l}}=0 ; \forall k, j=1,2, \ldots, n(26)
$$

Note that being constant the elements of the matrix $u_{j l}$, the Hamiltonian (18) is only valid for those cases that the matrix $u_{j l}$ holds (26). Take into account in (26) that the cases $k=j$ hold identically, thus the cases $k<j$ are the unique ones to consider because the cases $k>j$ provide the same particular equations than $k<j$. This outcome is easy to check by exchanging the subscripts $k \leftrightarrow j$ in (26). Such as it is shown in Section 6, Equation (26), and therefore the Hamiltonian (18), is always valid for the onedimensional $(n=1)$ case, and for the $n \geq 1$ case of autonomous linear dynamical systems. 


\section{Second order Schrodinger equation and its probabilistic interpretation}

By assuming that (26) holds the Hamiltonian (18), the Schrodinger equation can be deduced by applying the corresponding quantization rules to (18) (Galindo \& Pascual, 1990). Note that the rule (8) provides for the second order in momenta of (18):

$$
\frac{1}{2} \sum_{j l} u_{j l} \hat{p}_{j} \hat{p}_{l} \Psi(t, \boldsymbol{q})=-\frac{\sigma^{2}}{2} \sum_{j l} u_{j l} \frac{\partial^{2} \Psi(t, \boldsymbol{q})}{\partial q_{j} \partial q_{l}}(27)
$$

Considering (27) together (6)-(10), the second order Schrodinger equation deduced is:

$$
i \sigma \frac{\partial \Psi(t, \boldsymbol{q})}{\partial t}=-\frac{\sigma^{2}}{2} \sum_{j l} u_{j l} \frac{\partial^{2} \Psi(t, \boldsymbol{q})}{\partial q_{j} \partial q_{l}}-i \sigma \boldsymbol{f}(t, \boldsymbol{q}) \nabla \Psi(t, \boldsymbol{q})-i \frac{\sigma}{2}(\boldsymbol{\nabla} \boldsymbol{f}(t, \boldsymbol{q})) \Psi(t, \boldsymbol{q})(28)
$$

For the case of autonomous systems the functions $\boldsymbol{f}(t, \boldsymbol{q})=\boldsymbol{f}(\boldsymbol{q})$, and the change:

$$
\Psi(t, \boldsymbol{q})=\mathrm{e}^{-i \frac{E}{\sigma} t} \psi(\boldsymbol{q})(29)
$$

can be done in (28), being $E$ the system energy (22):

$$
\frac{\sigma^{2}}{2} \sum_{j l} u_{j l} \frac{\partial^{2} \psi(\boldsymbol{q})}{\partial q_{j} \partial q_{l}}+i \sigma \boldsymbol{f}(\boldsymbol{q}) \boldsymbol{\nabla} \psi(\boldsymbol{q})+\left(i \frac{\sigma}{2} \boldsymbol{\nabla} \boldsymbol{f}(\boldsymbol{q})+E\right) \psi(\boldsymbol{q})=0(30)
$$

Equation (30) is the time-independent second order Schrodinger equation, always valid for autonomous dynamical systems that hold (26). Note that conversely to the natural systems this equation is complex, not real.

The probabilistic interpretation of (28) can be done again through the split of the wave function in the polar coordinates (12). Its substitution in (28) provides, respectively for the real and the imaginary parts of the equation (after cancelling the term $\mathrm{e}^{i \frac{\mathrm{B}(t, \boldsymbol{q})}{\sigma}}$ ):

$$
\begin{gathered}
-\mathrm{A}(t, \boldsymbol{q}) \frac{\partial \mathrm{B}(t, \boldsymbol{q})}{\partial t}=\frac{\mathrm{A}(t, \boldsymbol{q})}{2} \sum_{j l} u_{j l} \frac{\partial B(t, \boldsymbol{q})}{\partial q_{j}} \frac{\partial B(t, \boldsymbol{q})}{\partial q_{l}}+\mathrm{A}(t, \boldsymbol{q}) \boldsymbol{f}(t, \boldsymbol{q}) \boldsymbol{\nabla B}(t, \boldsymbol{q})-\frac{\sigma^{2}}{2} \sum_{j l} u_{j l} \frac{\partial^{2} \mathrm{~A}(t, \boldsymbol{q})}{\partial q_{j} \partial q_{l}}(31) \\
-\sigma \frac{\partial \mathrm{A}(t, \boldsymbol{q})}{\partial t}= \\
-\sigma \boldsymbol{f}(t, \boldsymbol{q}) \boldsymbol{\nabla} \mathrm{A}(t, \boldsymbol{q})-\frac{\sigma}{2} \mathrm{~A}(t, \boldsymbol{q})(\boldsymbol{\nabla} \boldsymbol{f}(t, \boldsymbol{q}))-\sigma \sum_{j l} u_{j l} \frac{\partial A(t, \boldsymbol{q})}{\partial q_{j}} \frac{\partial B(t, \boldsymbol{q})}{\partial q_{l}}--\mathrm{A}(t, \boldsymbol{q}) \frac{\sigma}{2} \sum_{j l} u_{j l} \frac{\partial^{2} \mathrm{~B}(t, \boldsymbol{q})}{\partial q_{j} \partial q_{l}}(32)
\end{gathered}
$$

Dividing (31) by $\mathrm{A}(t, \boldsymbol{q})$ and dividing (32) by $\sigma$ and subsequently multiplying it by $2 \mathrm{~A}(t, \boldsymbol{q})$ : 


$$
\begin{gathered}
\frac{\partial \mathrm{B}(t, \boldsymbol{q})}{\partial t}+\frac{1}{2} \sum_{j l} u_{j l} \frac{\partial B(t, \boldsymbol{q})}{\partial q_{j}} \frac{\partial B(t, \boldsymbol{q})}{\partial q_{l}}+\boldsymbol{f}(t, \boldsymbol{q}) \boldsymbol{\nabla} B(t, \boldsymbol{q})-\frac{\sigma^{2}}{2 \mathrm{~A}(t, \boldsymbol{q})} \sum_{j l} u_{j l} \frac{\partial^{2} \mathrm{~A}(t, \boldsymbol{q})}{\partial q_{j} \partial q_{l}}=0 \\
\frac{\partial \mathrm{A}^{2}(t, \boldsymbol{q})}{\partial t}+\boldsymbol{\nabla}\left[(\boldsymbol{f}(t, \boldsymbol{q})+\boldsymbol{F}(t, \boldsymbol{q})) \mathrm{A}^{2}(t, \boldsymbol{q})\right]=0
\end{gathered}
$$

Equation (34) represents the law of probability conservation with current density $\boldsymbol{J}(t, \boldsymbol{q})$ :

$$
\boldsymbol{J}(t, \boldsymbol{q})=\boldsymbol{f}(t, \boldsymbol{q})+\boldsymbol{F}(t, \boldsymbol{q})(35)
$$

with components:

$$
J_{j}(t, \boldsymbol{q})=f_{j}(t, \boldsymbol{q})+F_{j}(t, \boldsymbol{q})=f_{j}(t, \boldsymbol{q})+\sum_{l} u_{j l} \frac{\partial B(t, \boldsymbol{q})}{\partial q_{l}}(36)
$$

Besides, (33) is the Hamilton-Jacobi equation (21) plus a correction term $V(t, \boldsymbol{q})$ :

$$
V(t, \boldsymbol{q})=-\frac{\sigma^{2}}{2 \mathrm{~A}(t, \boldsymbol{q})} \sum_{j l} u_{j l} \frac{\partial^{2} \mathrm{~A}(t, \boldsymbol{q})}{\partial q_{j} \partial q_{l}}(37)
$$

\section{The mesoscopic approach to dynamical systems}

The term (37) provides the stochastic part of the second order quantum formulation that the first order quantum formulation does not provide. In fact, if this term is considered in the Hamiltonian, it becomes:

$$
H_{s}(t, \boldsymbol{q}, \boldsymbol{p})=\frac{1}{2} \sum_{j l} u_{j l} p_{j} p_{l}+\sum_{j} f_{j}(t, \boldsymbol{q}) \cdot p_{j}+V(t, \boldsymbol{q})=H(t, \boldsymbol{q}, \boldsymbol{p})+V(t, \boldsymbol{q})(38)
$$

which has as canonical equations:

$$
\begin{gathered}
\dot{q}_{k}=\frac{\partial H_{s}(t, \boldsymbol{q}, \boldsymbol{p})}{\partial p_{k}}=f_{k}(t, \boldsymbol{q})+\sum_{l} u_{k l} p_{l}(39) \\
\dot{p}_{k}=-\frac{\partial H_{s}(t, \boldsymbol{q}, \boldsymbol{p})}{\partial q_{k}}=-\sum_{j} \frac{\partial f_{j}(t, \boldsymbol{q})}{\partial q_{k}} p_{j}-\frac{\partial V(t, \boldsymbol{q})}{\partial q_{k}}(40)
\end{gathered}
$$

Developing now the same operations (24) and (25) on (39) and (40), and considering that (26) holds:

$$
\ddot{q}_{k}(t)=\frac{\partial f_{k}(t, \boldsymbol{q})}{\partial t}+\sum_{l} \frac{\partial f_{k}(t, \boldsymbol{q})}{\partial q_{l}} f_{l}(t, \boldsymbol{q})-\sum_{l} u_{k l} \frac{\partial V(t, \boldsymbol{q})}{\partial q_{l}}(41)
$$


Equation (41) represents the mesoscopic approach to the dynamical system (17). Obviously it is considered like this because $V(t, \boldsymbol{q})$ depends on $\mathrm{A}(t, \boldsymbol{q})$, i.e., on the square root of the probability density.

If the time integral is taken at both sides of (41):

$$
\dot{q}_{k}(t)=f_{k}(t, \boldsymbol{q})-\sum_{l} u_{k l} \int_{t_{0}}^{t} \frac{\partial V(t, \boldsymbol{q})}{\partial q_{l}} d t(42)
$$

Equation (42) is the mesoscopic approach saught for the general systems (1) under the assumption of (26). This mesoscopic formulation is the opposite one to the followed for the stochastic differential equations known as Ito equations and the Fokker-Planck equation (Haken, 2004). In that approach, the Ito equations present the stochastic terms in advance, i.e.:

$$
\dot{q}_{k}(t)=f_{k}(t, \boldsymbol{q})+\sum_{l} u_{k l} w_{l}(t)(43)
$$

where $u_{k l}$ are constant known elements and $w_{l}(t)$ are the stochastic terms, which have a mathematical white noise structure, i.e.:

$$
\left\langle d w_{l}(t)\right\rangle=0 ;\left\langle d w_{l}(t), d w_{m}(t)\right\rangle=\delta_{l m} d t(44)
$$

In (44) " $<>$ " represents the time average. From the Ito equations (43) and from (44) the Fokker-Planck equation can be deduced (Haken, 2004):

$$
\frac{\partial \rho(t, \boldsymbol{q})}{\partial t}+\nabla[(\boldsymbol{f}(t, \boldsymbol{q})+\boldsymbol{F}(t, \boldsymbol{q})) \rho(t, \boldsymbol{q})]=0
$$

where $\rho(t, \boldsymbol{q})$ is the probability density. Equation (45) represents also the law of probability conservation with current density $\boldsymbol{J}(t, \boldsymbol{q})$ :

$$
\boldsymbol{J}(t, \boldsymbol{q})=\boldsymbol{f}(t, \boldsymbol{q})+\boldsymbol{F}(t, \boldsymbol{q})(46)
$$

with components:

$$
J_{j}(t, \boldsymbol{q})=f_{j}(t, \boldsymbol{q})+F_{j}(t, \boldsymbol{q})=f_{j}(t, \boldsymbol{q})-\frac{Q}{2} \sum_{l} \sum_{m} u_{j m} u_{m l} \frac{\partial \ln (\rho(t, \boldsymbol{q}))}{\partial q_{l}}(47)
$$


Equation (47) has a more general structure if $u_{k l}=u_{k l}(t, \boldsymbol{q})$. However, (47) is enougth to compare it with (34). In addition, $Q$ is the so-called diffusion parameter. Observe that if the equivalence $\rho(t, \boldsymbol{q}) \rightarrow$ $\mathrm{A}^{2}(t, \boldsymbol{q})$ is assumed, the difference between (34) and (47) is present in the vector $\boldsymbol{F}(t, \boldsymbol{q})$, which in the Fokker-Planck equation depends on $\rho(t, \boldsymbol{q}) \rightarrow \mathrm{A}^{2}(t, \boldsymbol{q})$, but not on the generalized action $B(t, \boldsymbol{q})$.

The connection between both formulations could be hypothetically appreciated if the term $B(t, \boldsymbol{q})$ could be put in function of $A(t, \boldsymbol{q})$ by (33) and substituted in (34). Even if this substitution was able to be done as an approximated calculation, the connection could be stated. Also the relationship between the system Planck constant $\sigma$ and the diffusion parameter $Q$ could be investigated. By the moment, these hypotheses have not been solved yet.

\section{Application cases}

To obtain the second order Schrodinger equation (28) for the application cases, the method consist in getting, if possible, the values $u_{j l}$ that hold (26). If the system is autonomous, the equation considered is the time-independent second order Schrodinger equation (30).

Two cases are here considered that hold (26): the one-dimensional case, trying to solve the autonomous case and its particular linear case, and the linear autonomous case for an arbitrary dimension.

\subsection{The one-dimensional case}

Note that if $n=1,(26)$ holds trivially. If for this case the subscripts are avoided by being unnecessary. The Hamiltonian (18) can be written as:

$$
H(t, q, p)=\frac{1}{2} u p^{2}+f(t, q) p(48)
$$

The value of the parameter $u$ in (48) is non-determined in advance. The corresponding second order Schrodinger equation is:

$$
i \sigma \frac{\partial \Psi(t, q)}{\partial t}=-\frac{\sigma^{2}}{2} u \frac{\partial^{2} \Psi(t, q)}{\partial q^{2}}-i \sigma f(t, q) \frac{\partial \Psi(t, q)}{\partial q}-i \frac{\sigma}{2} \frac{\partial f(t, q)}{\partial q} \Psi(t, q)
$$

If the system is autonomous, the system energy is:

$$
E=\frac{1}{2} u p^{2}+f(q) p(50)
$$

and the time-independent second order Schrodinger equation:

$$
\frac{\sigma^{2}}{2} u \psi^{\prime \prime}(q)+i \sigma f(q) \psi^{\prime}(q)+\left(i \frac{\sigma}{2} f^{\prime}(q)+E\right) \psi(q)=0(51)
$$


The change:

$$
\psi(q)=\mathrm{e}^{-\frac{i}{u \sigma} \int f(q) d q} \Omega(q)
$$

provides, after some calculations, the differential equation:

$$
\Omega^{\prime \prime}(q)+\left(\frac{f^{2}(q)}{u^{2} \sigma^{2}}+\frac{2 E}{u \sigma^{2}}\right) \Omega(q)=0(53)
$$

Note that although (53) seems a real differential equation, their solutions can be complex. For autonomous nonlinear systems, for instance the logistic function case $f(q)=a q\left(1-\frac{q}{b}\right)$, the search ot its solutions represents by itself an open investigation field. For instance, for a complete handbook of differential equations such as (Polyanin \& Zaitsev, 2002), no solutions are found for this case. Note as well that the exact solution is necessary to find the possible eigenvalues as energy levels and the corresponding eigenfunctions.

However, if the function is linear, then $f(q)=\alpha_{0}+\alpha q$. However, the linear functions are not interesting in the applications except if they are a first order approximation to the nonlinear ones about a steady state $q=Q$, for which $f(Q)=0$.

The first order approximation of a nonlinear function with a steady state $q=Q$ about this state is $f(q) \cong f(Q)+f^{\prime}(Q)(q-Q)=-f^{\prime}(Q) Q+f^{\prime}(Q) q$. The change $x=-f^{\prime}(Q) Q+f^{\prime}(Q) q$ and $\bar{\Omega}(x)=$ $\Omega\left(q=\frac{x+f^{\prime}(Q) Q}{f^{\prime}(Q)}\right)$ can be done in $(53)$ and it becomes:

$$
\bar{\Omega}^{\prime \prime}(x)+\left(\beta_{1}^{2} x^{2}+\beta_{0}\right) \bar{\Omega}(x)=0 ; \beta_{1}=\frac{1}{u f^{\prime}(Q) \sigma} ; \beta_{0}=\frac{2 E}{u\left(f^{\prime}(Q) \sigma\right)^{2}}(54)
$$

The solutions of (54) are a linear combination of the Parabolic-Cylinder functions (see Equation 2.1.2-1.1 of Polyanin \& Zaitsev, 2002). However, by the functions DSolve and Expand of MATHEMATICA-10.2 the solution can be put in function of the Hermite functions $H(v, \mathrm{y})$, wich become (complex or real) polynomials if the parameter $v$ is an integer. The final outcome, after multipliying by the complex exponential of (52) and definining $\bar{\psi}(x)=\psi\left(q=\frac{x+f^{\prime}(Q) Q}{f^{\prime}(Q)}\right)$, is:

$$
\begin{aligned}
& \bar{\psi}(x)=\mathrm{e}^{-i \beta_{1} \frac{x^{2}}{2}} \bar{\Omega}(x)= \\
& =k_{1} \mathrm{e}^{-i \beta_{1} x^{2}} 2^{\frac{-i \beta 0+\beta 1}{4 \beta 1}} H\left(-\frac{1}{2}+\frac{i \beta 0}{2 \beta 1}, \frac{-1+i x \sqrt{\beta 1}}{\sqrt{2}}\right)+k_{2} 2^{\frac{-i \beta 0+\beta 1}{4 \beta 1}} H\left(-\frac{1}{2}+\frac{i \beta 0}{2 \beta 1}, \frac{-1+i x \sqrt{\beta 1}}{\sqrt{2}}\right) \\
& \psi(q)=\bar{\psi}\left(f(Q)+f^{\prime}(Q)(q-Q)\right) ; \beta_{1}=\frac{1}{u f^{\prime}(Q) \sigma} ; \beta_{0}=\frac{2 E}{u\left(f^{\prime}(Q) \sigma\right)^{2}}(56)
\end{aligned}
$$


Equations (55) and (56) represent the general solution of the time-independent wave function, being $k_{1}$ and $k_{2}$ in (55) arbitrary complex constants. Note that the parameter $v=-\frac{1}{2}+\frac{i \beta 0}{2 \beta 1}$ is not an integer in both Hermite functions, thus neither the condition of the system energy quantization nor the convergence of the wave function in $q \rightarrow \pm \infty$ is provided. Therefore, if concrete values of $k_{1}$ and $k_{2}$ in (55) permit the quantization of the system energy and the convergence of the wave function in $q \rightarrow \pm \infty$ is still under investigation. However, a hypothesis hold is that this research could be developed on the square wave function $\Psi^{2}(t, q)=\psi^{2}(q)$ rather than on the function $\psi(q)$.

\subsection{The autonomous linear multidimensional case}

An autonomous linear multidimensional system is a particular case of (1), which takes the following formula:

$$
\dot{q}_{k}(t)=a_{0}+\sum_{j} a_{k j} q_{j}(57)
$$

Note in (57) that $a_{k j}$ is not a simetric matrix and it is a generalization to $n \geq 1$ dimensions of the linear case studied in Section 6.1. In fact, similarly to that case, the interesting case is the study of the linear approximation to a nonlinear case about a steady state $\boldsymbol{q}=\boldsymbol{Q}$, for which $\boldsymbol{f}(\boldsymbol{Q})=\mathbf{0}$. If in (1) a first order approximation about $\boldsymbol{q}=\boldsymbol{Q}$ is done:

$f_{k}(\boldsymbol{q}) \cong f_{k}(\boldsymbol{Q})+\left.\sum_{j} \frac{\partial f_{k}(\boldsymbol{q})}{\partial q_{j}}\right|_{\boldsymbol{q}=\boldsymbol{Q}}\left(q_{j}-Q_{j}\right)=-\left.\sum_{j} \frac{\partial f_{k}(\boldsymbol{q})}{\partial q_{j}}\right|_{\boldsymbol{q}=\boldsymbol{Q}} Q_{j}+\left.\sum_{j} \frac{\partial f_{k}(\boldsymbol{q})}{\partial q_{j}}\right|_{\boldsymbol{q}=\boldsymbol{Q}} q_{j}(58)$

And comparing (57) and (58):

$$
a_{0}=-\left.\sum_{j} \frac{\partial f_{k}(\boldsymbol{q})}{\partial q_{j}}\right|_{\boldsymbol{q}=\boldsymbol{Q}} Q_{j} ; a_{k j}=\left.\frac{\partial f_{k}(\boldsymbol{q})}{\partial q_{j}}\right|_{\boldsymbol{q}=\boldsymbol{Q}}
$$

Note moreover that for an autonomous linear multidimensional system (57) and (58), from (59) can be deduced that:

$$
\frac{\partial f_{k}(t, \boldsymbol{q})}{\partial q_{j}}=a_{k j}=\left.\frac{\partial f_{k}(\boldsymbol{q})}{\partial q_{j}}\right|_{\boldsymbol{q}=\boldsymbol{Q}}(60)
$$

Thus the conditions (26) become for this case:

$$
\sum_{l} u_{j l} a_{k l}-\sum_{l} u_{k l} a_{j l}=0 ; \forall k, j=1,2, \ldots, n(61)
$$


In (61) $a_{k l}$ are known constants by (60), thus (61) will hold generally.

The concrete application case chosen is the Van der Pol oscillator, with $x$ as oscillating variable and $\varepsilon$ as parameter:

$$
\ddot{x}(t)-\varepsilon\left(1-x^{2}\right) \dot{x}(t)+x(t)=0(62)
$$

By doing the changes $q_{1}=\dot{x}$ and $q_{2}=x,(62)$ becomes the two-dimensional dynamical system:

$$
\left.\begin{array}{c}
\dot{q}_{1}(t)=-q_{2}(t)+\varepsilon\left(1-q_{2}^{2}(t)\right) q_{1}(t) \\
\dot{q}_{2}(t)=q_{1}(t)
\end{array}\right\}
$$

The only steady state of $(63)$ is $\boldsymbol{Q}=\left(Q_{1}, Q_{2}\right)=(0,0)$. The linearization about $\boldsymbol{Q}$ is:

$$
\left.\begin{array}{c}
\dot{q}_{1}(t)=f_{1}\left(q_{1}, q_{2}\right)=\varepsilon q_{1}(t)-q_{2}(t) \\
\dot{q}_{2}(t)=f_{2}\left(q_{1}, q_{2}\right)=q_{1}(t)
\end{array}\right\}
$$

Thus, from (60) and (64), the elements $a_{k l}$ of (60) are: $a_{11}=\varepsilon, a_{12}=-1, a_{21}=1$ and $a_{22}=0$. And (61) provides for $k=1$ and $j=2$ the equation:

$$
u_{12} a_{11}+u_{22} a_{12}=u_{11} a_{21}+u_{12} a_{22}(65)
$$

Note in (65) that the case $j=1$ and $k=2$ provides the same equation, and that the cases $j=k$ hold identically. In addition, considering that $u_{21}=u_{12}$, the outcome equation is:

$$
u_{11}-\varepsilon u_{12}+u_{22}=0(66)
$$

A solution of (66) is $u_{12}=u_{22}=1$ and $u_{11}=\varepsilon-1$. Then, the time-independent second order differential equation can be written as:

$$
\begin{aligned}
\sigma^{2}\left(\frac{(\varepsilon-1)}{2} \frac{\partial^{2} \psi(\boldsymbol{q})}{\partial q_{1}^{2}}+\frac{\partial^{2} \psi(\boldsymbol{q})}{\partial q_{1} \partial q_{2}}\right. & \left.+\frac{1}{2} \frac{\partial^{2} \psi(\boldsymbol{q})}{\partial q_{2}^{2}}\right)+i \sigma\left(\left(\varepsilon q_{1}-q_{2}\right) \frac{\partial \psi(\boldsymbol{q})}{\partial q_{1}}+q_{1} \frac{\partial \psi(\boldsymbol{q})}{\partial q_{2}}\right)+ \\
& +\left(i \frac{\sigma \varepsilon}{2}+E\right) \psi(\boldsymbol{q})=0(67)
\end{aligned}
$$


Actuallly, even for a linear two-dimensional case, the corresponding time-independent second order differential equation (67) is complex to be solved. In this case, as well as in similar cases, the search of the solution is an open research by the moment.

\section{Discussion and conclusions}

This paper is an attempt to state an isomorphism between natural laws in physics and general systems. It is done by translating the quantum theory from the first field to the second one. It must be valued taking into account the works (Micó, 2014a) and (Micó, 2014b) together the present paper. The three papers together go beyond the derivation of a Schrodinger equation for general systems. It is like this because they try as well to state an analytical formalism for general systems, i.e., a Lagrangian-Hamiltonian approach plus a study of the Hamilton-Jacobi equation, and what are the roles that these approaches play to interpret the quantum theory provided.

Note that the formalism provided is still an open research. First of all it is correct under some assumptions and, besides, it just arrives until some unsolved points. The following paragraphs try to discuss these assumptions and points.

The first assumption is the second order structure of the Hamiltonian to reproduce the equations of the general systems in Section 3. Although there could be other options, it is taken from the physics in order to obtain a mesoscopic approach that a first order Hamiltonian of Section 2 does not provide.

Note that the second order Hamiltonian (18) also assumes that the functions $u_{j l}$ must be constant. In addition, this Hamiltonian is correct if Equations (26) hold. All the subsequent derivations of the formalism also depend on the fact that (26) must be held. Fortunately, the one-dimensional and the linear autonomous cases hold Equations (26), such as it is proved in Section 6. If for some other cases Equations (26) holds, such as nonlinear multidimensional cases with dimension greater than one, it should be checked for the particular cases under study. For instance, the author has checked that the nonlinear case of dimension two given by the predator-prey system does not hold (26). Due to, neither the second order Hamiltonian (18) nor the second order Schrodinger equation (28) can be applied for this case.

The way about how to generalize the second order Hamiltonian (18) to be correct for any dynamical system (1) redefined as the second order form (17) is being investigated by the author. One way sketched is considering that the constants $u_{j l}$ become functions $u_{j l}(t, \boldsymbol{q})$. In fact, the author has checked that similar but more complex structures can be derived for the Hamilton-Jacobi equation (33) and for the probability conservation law (34) although the functions $u_{j l}(t, \boldsymbol{q})$ become unknown. In addition, they also present a similarity to the Fokker Planck equation (45) when the constant stochastic values $u_{k l}$ of (43) become functions $u_{k l}(t, \boldsymbol{q})$ (Haken, 2004). However, by the moment no definitive conclusions about the mathematical structure of these functions haven been obtained.

Other ways to overcome the first order approaches of Section 2 could be considered in a future researches. A way is the consideration of a canonical transformation on (2) that provides a second order Hamiltonian. An alternative way is considering the addition of a term in (2) that contains the momenta elevated to a fractional exponent between one and two, in such a way that the fractional calculus should be used to develop the quantization rules.

Other problem to be commented is that provided in (Micó, 2014b) about the meaning of the system Planck constant in the case of general systems. In that work was said that the system Planck constant is particular of each system under description. The point of view defended is that it represents the mathematical limits of the system knowledge. However, the most probable is that some experiments must 
be designed to find the particular values of these constants. The hypothesis about the nature of this constant continues being the same. The existence of a diffusion parameter in the context of the FokkerPlanck equation (45)-(47) own of each system strengthens this argument. Another question is if both constants are related in some way. Finding the actual relationship between both formalisms, the quantum approach here presented and the corresponding to the Fokker-Planck equation should be also a future strength of research.

Finally, the open questions of the theory presented must be investigated. First of all, the solution (55)-(56) is not acceptable for a one-dimensional linear autonomous system, unless a suitable combination of the two independent functions provides the eigenvalues for the system energy and the corresponding eigenfunctions. Another open question is if this problem happens only with linear systems and for nonlinear systems the eigenvalues and eigenfunctions arise. However, note that getting solutions of a nonlinear problem such as (51)-(53) is a great problem by itself. The same conclusion can be deduced when the system has a dimension greater than one, although the system is linear, such as it happens in (67): getting the solution of that partial differential equation is by itself a problem of great complexity.

\section{References}

DIRAC, PAUL A. M. (1964). Lectures on Quantum Dynamics. New York. Belfer Graduate School of Science, Yeshiva University.

GALINDO, A. \& PASCUAL, P. (1990). Quantum Mechanics I \& II. Berlin-Heidelberg-New York. Springer-Verlag.

HAKEN H. (2004). Synergetics. Introduction and Advanced Topics. Berlin-Heidelberg. Springer-Verlag.

HAVAS, P. (1973). "The conection between Conservation laws and Invariance Groups:

Folklore, Fiction, and Fact" en Acta Physica Austriaca vol. 38, p. 145-167.

MICÓ, J. C. (2014a). "An analytical formalism of dynamical systems ". In 9th Congress of the EUS-UES. Globalization and Crisis; Systems; Complexity and governance. Ciutat de Valéncia: ADD editorial. 501-510.

MICÓ, J. C. (2014b). "A quantum formalism of dynamical systems". In 9th Congress of the EUS-UES. Globalization and Crisis; Systems; Complexity and governance. Ciutat de Valéncia: ADD editorial. 511-518.

POLYANIN, A. D. \& ZAITSEV, F. Z. (2002). Handbook of Exact Solutions for Ordinary Differential Equations (second edition). Londo-New York-Washington, D.C. Chapman and Hall/CRC.

PONTRYAGIN, L. S. (1985). The Mathematical Theory of Optimal Processes. New York.

Gordon and Breach Science Publishers. 